\title{
How do health professionals decide whether an interpreter is needed for families in neonatal and pediatric units?
}

Liz Jones ${ }^{\mathrm{a}^{*}}$, Nicola Sheeran ${ }^{\mathrm{a}}$, Rachyl Pines ${ }^{\mathrm{b}}$ and Bradley Saunders ${ }^{\mathrm{a}}$

${ }^{a}$ School of Applied Psychology, Griffith University, Mt Gravatt, AUSTRALIA

${ }^{\mathrm{b}}$ Department of Communication, University of California Santa Barbara, USA.

* Corresponding Author: School of Applied Psychology, Griffith University, 176 Messines Ridge Rd, Mt Gravatt, QLD 4122 AUSTRALIA

E: 1.jones@griffith.edu.au

The authors report no conflicts of interest. 


\section{Highlights}

Health professionals find it difficult to decide whether an interpreter is needed

Health professionals' attitudes and goals affect their decision making about interpreters

There is marked variability in the decision-making process individual health professionals use

Training is needed, including a decision tool, to assist health professionals' decisions

Objective: To examine how health professionals decide whether family members require an interpreter.

Methods: 69 health professionals, doctors, nurses, and allied health, from neonatal and pediatric units participated. Interviews used a verbal protocol analysis, which elicited their thoughts about using interpreters, including how they decided if an interpreter was needed.

Results: Five themes captured the decision-making process health professionals use. Of these, three themes described the goals and beliefs participants brought to their interactions with family members: Ensuring understanding, Addressing socioemotional needs, and Who decides. The theme Assessing understanding was prominent within the interaction, while the final theme was Contextual factors influencing decision making. No differences were found between mono and multilingual participants, and few differences between health professional groups.

Conclusion: Health professionals find it difficult to assess whether a family member needs an interpreter and there is no consistency in how they make this decision, with some using heuristics and others a more systematic approach. Health professionals have beliefs about the purpose of an interpreter that potentially limit the voice of family members.

Practice Implications: Health professionals need training to assist them in decisions about whether an interpreter is needed, including a decision tool and knowledge about policies.

Keywords: health communication; interpreter; decision-making 
How do health professionals decide when an interpreter is needed?

\section{Introduction}

Optimal health service delivery in hospitals depends in part on effective communication with patients and families, particularly in neonatal and paediatric units, where family-centred models of care (FCC) require collaborative relationships between staff and parents to care for child patients $[1,2]$. Although medical care of the child is the primary focus, health professionals (HPs) are expected to involve parents in decision making and their infant's care $[1,3]$. Thus, staff use communication to empower parents, provide information about the child's progress, and build relationships [2, 4, 5]. However, for HPs engaging in FCC with families with low host language proficiency (LHLP), language and other cultural differences are barriers to FCC [6-8], with implications for quality of health care, including culturally competent care [8]. This is an increasing problem in Australia (and other countries), as numbers of international tourists, immigrants, and refugees with LHLP increase [9]. To facilitate communication with LHLP families, HPs often rely on interpreters. However, little is known about how HPs decide whether a family requires an interpreter, which is the focus of the current study.

\subsection{Communicating with LHLP patients and families}

HPs experience difficulties in communication with LHLP patients in general [10], and with LHLP families in neonatal and paediatric units specifically $[8,11-13]$. Nurses describe having less communication, less informal communication such as chatting, more intergroup communication, and more focus on ensuring parents understood information with LHLP families [13]. This is inconsistent with parent preferences for nurse communication [4, 14], although LHLP families in neonatal and paediatric units' preferences have not been examined, although a study in cancer care found migrant groups differed in their communication preferences [15]. 
To facilitate communication with LHLP patients and families, HPs may use interpreters. Indeed, in many countries, including Australia [16], interpreter use is mandated, with policies outlining when an interpreter should be used and who can be used as an interpreter. When communicating with LHLP patients, HPs may use various interpreters, including professional interpreter services (in-person or telephone), bilingual staff members, and family members, however in our country professional interpreters are mandated in most situations.

The utilization of professional interpreters improves patients' and families' satisfaction with clinicians and clinical care [17-20]. A systematic review found professional interpreters raised the quality of care of LHLP patients to a level similar to patients without language barriers, and improved care more than ad-hoc (i.e., bilingual staff/family) interpreters [21]. Professional interpreter utilization may also reduce health service costs. While a Swiss study found utilizing interpreters meant higher short-term health costs for those with language barriers [22], a study of physicians in a paediatric emergency department (ED), found treatment was most cautious and expensive for LHLP patients when no interpreter was utilized [12]. Relatedly, Jacobs et al [23] found utilization of interpreters in ED did not increase long-term costs of patient care, because interpreter costs were offset by decreased costs from the subsequent reduction in ED visits for those who had an interpreter. However, not all studies find benefits of interpreters. For example, Ngo-Metzger et al [24] found utilizing interpreters for patients with language discordant providers made interpersonal care (e.g., respect and adequate explanation) worse.

Despite researchers and policy-makers advocating for utilization of interpreters, there is extensive evidence of under-utilization of professional interpreters in health care. Queensland Health [25] reported nearly half of clients with LHLP did not receive an interpreter each time they visited a facility, despite legislation mandating utilization of 
professional medical interpreters. A US study similarly found in EDs only $69 \%$ of LHLP patients had any interpreter [26], and another US study found interpreters were utilized only $60 \%$ of the time with physicians and $37 \%$ with nurses [27]. Nurses rarely utilize interpreters, even when professional interpreter services are available, and despite recognizing the benefits of interpreter services [28]. Instead, HPs report "making do" by utilizing ad-hoc interpreters. There is some utilization of bilingual staff (10\% in [26]), however family members are utilized more frequently [26, 28-31]. Moreover, contrary to most hospital policies, one study found for $20 \%$ of medical residents utilizing child interpreters was not rare [31]. Doctors recognise they under-utilize professional interpreters, weighing the value for clinical decision-making against their time constraints, and the availability and poor quality of interpreter services, with family members or staff members seen as more convenient $[18,28]$. Doctors normalise under-utilisation, despite acknowledging it influences patients' care [18]. There is also evidence patients and family members may be satisfied with or even prefer family member interpreters [32].

In summary, interpreters, particularly professional interpreters, generally improve clinical care, yet are under-utilized. Some research has demonstrated reasons for this underutilization (e.g., time constraints, norms). However, what has not been examined is how HPs decide whether an interpreter, either professional or ad hoc, is needed or not, specifically with families in neonatal or paediatric units, where communication extends to FCC. Yet incorrect decisions are consequential - not accessing an interpreter when one is needed affects patient satisfaction, clinical outcomes, and financial costs, and accessing an interpreter when one is not needed is costly. Our study examined HPs' decision-making processes about whether family members need an interpreter.

\subsection{Theoretical approach}


Our study was informed by the Heuristic-Systematic processing model of decision making (HSM) [33]. HSM proposes individuals use two strategies to process information: heuristic and systematic processing [34]. Heuristic processing enables quick and efficient decisions, through reducing cognitive effort for the decision maker, by using a subset of the available information and simple decision rules [34]. There are multiple heuristics people (including HPs) may use [35, 36]: satisficing, where HPs decide when they can provide good enough care without an interpreter; fast and frugal evaluations, where HPs makes decisions quickly and with limited information, such as "Can the person answer my questions?"; the availability heuristic, which relies on immediate examples that come to a person when evaluating another person (e.g., the last occasion when a HP needed an interpreter); and the representativeness heuristic, where a person is similar in essential characteristics to a HP's stereotype of a person with LHLP, e.g., skin colour or dress.

In contrast, systematic processing involves people making decisions by analysing information, hypothesis testing, and integrating thoughts and feelings. Systematic processing requires more cognitive effort [37], and is used more when making decisions with major consequences for either yourself or others [34]. Heuristic and systematic processing are not mutually exclusive and may occur interdependently, whereby systematic processing may become biased as the decision maker gathers or interprets information selectively, based on beliefs or attitudes [37]. For many years, systematic processing was thought to result in more accurate decision making. However, emerging evidence suggests heuristics are not only faster than a full appraisal of information, but may be more accurate than systematic decision-making in some contexts, including medical decision making [33]. Research has not examined whether HPs use heuristics or systematic processing more in their decision-making about interpreter use. In summary, our research question was "how do health professionals decide whether family members need an interpreter?". 


\subsection{Method}

\subsection{Context}

This study was conducted in a large tertiary hospital in Queensland, Australia with neonatal and paediatric units. The hospital is located where $36 \%$ of the population were born overseas and 55\% had one or both parents born overseas [38]. While many people born overseas are from English speaking countries, a significant number were born in non-English speaking countries, including China, Japan, India, and Philippines, and in $14.9 \%$ of households a non-English language is spoken [38]. The area is a significant overseas tourist destination. The hospital is required to follow the Queensland Health policy regarding interpreter use [16], which requires an accredited interpreter when information is for significant health and/or health outcomes, and prohibits the use of children under 18 as interpreters. In a medical emergency bilingual staff, followed by relatives or friends may be used.

\subsection{Participants}

Sixty-nine participants were recruited, and their demographics are presented in Table 1.

\section{INSERT TABLE 1 ABOUT HERE}

\subsection{Procedure}

This study formed part of a larger study on decision making about interpreter use. Ethical approval was obtained from the University and the Hospital and Health Service Human Research Ethics Committees. The research team recruited participants directly during regular visits to the hospital, via flyers distributed in the units, and via email, depending on the process requested by each unit. Recruitment continued until the interviewers agreed saturation had been reached, and a representative sample from neonatal and pediatric units, health professions, and monolingual and multilingual staff had participated. 
Three interviewers (the second and fourth authors, and a student, all psychology trained interviewers) conducted interviews in a quiet area within the hospital. Participants were given consent forms and a brief demographics questionnaire. Interviews were approximately 20 minutes in length and digitally audio-recorded. We used a verbal protocol analysis ("think aloud" method), a well-recognized technique for capturing ongoing or recently completed decision processes [39]. VPA elicits actual thoughts, not explanatory or descriptive responses [40], and avoids imposing structure on the participant's decisionmaking structure. The interview protocol is presented in Table $2^{1}$. Interviews were transcribed verbatim by research team members and a transcriber. Two interviewers checked the accuracy of the transcriptions.

\section{INSERT TABLE 2 ABOUT HERE}

\subsection{Analysis}

Analysis of the transcripts was undertaken using thematic analysis, based on Braun and Clarke's [41] guidelines. Salient themes were identified and coded inductively from the interview transcripts. The first and second authors (LJ and NS), psychologists with extensive experience in qualitative research, coded all transcripts. Initially they separately coded the same 5 interviews to develop a descriptive coding protocol. They then reviewed each other's coding repeatedly during coding of the remaining interviews. Grouping of themes was then undertaken by the first author, in consultation with the second author, until final themes were determined that captured the decision-making processes used by participants to decide whether an interpreter was needed. The remaining authors read all transcripts and reviewed the final themes. After all interviews had been coded, we re-examined participants' responses to the initial question (their first thoughts). We also examined whether the content of the

\footnotetext{
${ }^{1}$ The terms interpreter and translator were both used in interviews, as many health professionals use the terms interchangeably.
} 
themes differed by the unit the HP worked in, their health profession, and whether they were monolingual or multilingual.

\subsection{Findings}

Our analysis identified five themes, which together with the sub-themes and exemplar quotes are presented in Table 3. Three themes were identified describing goals and beliefs participants brought to their interactions: Ensuring understanding, Addressing socioemotional needs and Who decides?. Within the interaction participants stated they focused on Assessing understanding (fourth theme). Decision making was also influenced by the fifth theme, Contextual factors. There were no differences between monolingual and multilingual participants, or different units for any theme. Furthermore, Assessing understanding was the only theme where health professional groups differed.

\section{INSERT TABLE 3 ABOUT HERE}

\subsection{Ensuring understanding}

A key goal influencing decision making for most participants was Ensuring understanding. This theme had three sub-themes describing participants' beliefs about: Whose understanding, Understanding what, and How much understanding.

\subsubsection{Whose understanding?:}

In describing who needed to understand, most HPs stated parents need to understand the HP's message, suggesting they focused on the 1-way transmission of their information to the parent. A small number of HPs talked about parents being able to communicate with HPs, or HPs being able to understand parents. However, these responses were often to the question about the pros and cons of using an interpreter, rather than to earlier interview questions, suggesting that while two-way communication was beneficial, it was not the most salient reason for engaging an interpreter. 
In general, it was agreed the need for an interpreter did not extend to family members beyond the parents. Many HPs also spoke about which parent needed to understand. For some an interpreter was unnecessary if one parent was fluent in English, whereas for others an interpreter was important if one parent did not speak English fluently. The decision that both parents needed to understand was motivated by two different beliefs. Some HPs expressed the importance of both parents understanding. Others were motivated by perceptions of whether the parent proficient in English could be trusted to interpret for the other parent. The latter concern was mostly about fathers, and often referred to the cultural background of the father.

Finally, a few participants spoke about interpreters only being necessary for doctors, rather than for other health professions.

\subsubsection{Understanding what?}

For many HPs understanding was mostly about parents understanding important or serious information, which was compared to "simple" or "small" information. There were a variety of descriptions of what was important information, including prognosis, end of life, discharge, medical history, and acute medical crises. Understanding was particularly important for parents being able to give informed consent. However, for nearly all HPs, important or serious information did not include non-medical information, discussions about how parents provided care, such as breastfeeding, or shared decision making. A minority of HPs also spoke about interpreters enabling understanding of culturally appropriate medical practices.

\subsubsection{How much understanding?:}

HPs varied as to what level of understanding was needed. Some HPs stated complete or full understanding was important. In contrast, others stated parents needed to understand "enough". There was little description about how someone could determine what "enough" 
understanding was, except that it included parents being able to consent and "important" information.

\subsection{Addressing socioemotional needs}

A goal for a smaller number of HPs was needing an interpreter to ensure parents felt heard; so that parents could raise concerns, discuss emotions, and feel comforted. This, in turn, was related to reducing parental distress. For example, one health professional described the comfort of hearing someone speak in their own language, and a small number talked about an interpreter allowing cultural sensitivity, and this being important for providing culturally appropriate communication.

\subsection{Who decides?}

Queensland Health policy states any health professional, or a patient or family member can request an interpreter. The majority of HPs believed the health professional should decide if an interpreter was needed. However, while most allied health and nursing participants believed they should make the decision, others talked about the decision being the responsibility of the doctor or a manager. Moreover, most participants spoke about individual HPs making the decision. However, a small number of nurses, and some allied health participants, regarded the decision as one based on the assessment of two or more staff.

Very few participants described asking parents if they wanted or needed an interpreter. Indeed, some HPs did not believe the request by some parents for an interpreter was legitimate. It appeared parents' preferences were mainly considered when they refused the offer of an interpreter.

\subsection{Assessing understanding}

The next theme, Assessing understanding, describes how within interactions HPs needed to assess a person's understanding. This is in addition to information in the patient's 
notes or from other HPs. Many HPs spoke about it being difficult to assess understanding. HPs detailed a range of factors affecting assessments of understanding, including: people may say they understand but they don't, stress reduces a person's competency in both comprehension and expression, and people may not admit they don't understand.

In assessing a person's understanding some HPs used a heuristic decision-making approach, whereas others engaged in more systematic decision making, and some used both. Nurses were more likely than other health professions to use heuristics, although many nurses also used a systematic approach.

The first way of assessing understanding was the person's membership of particular social categories. Those using this heuristic typically used one or more terms such as born overseas (or immigrant, refugee, tourist) or their appearance (skin colour, headwear, or dress). Some HPs noted this could be combined with a second demographic factor, such as their education level or gender (where women were assumed to be less fluent). Other HPs simplistically based their assessment of who needed an interpreter on did the person speak English or did they understand. Some went on to say that they would also find out how long the parent had been in Australia or what language they spoke at home.

Alternatively, or sometimes in addition, HPs engaged in a more systematic process of assessing the verbal and/or nonverbal behavior of the parent/s. This assessment could involve a small range of behaviors or could be quite complex. For example, a number of participants spoke about how the family members responded to questions. Some combined social categories with a verbal assessment based on missing words. A complex assessment could involve multiple steps and indicators of understanding, including facial expressions, verbal and non-verbal responses, expressive and receptive language skills, and use of simple to complex questions and answers. Some HPs talked about the importance of specifically 
assessing fluency in understanding medical jargon, as a person might reasonably understand English for everyday situations, but need medical information translated.

\subsection{Contextual factors}

The final theme describes a range of Contextual factors that influenced participants. Participants' beliefs about the excessive cost and lack of availability of interpreters influenced their decision making. Time also influenced their decisions. Participants talked about both being unable to wait due to the urgency of providing care, and whether their workload allowed time to organise an interpreter. It is noteworthy participants rarely described hospital policies and procedures about interpreter use informing their decision making. Indeed, most participants expressed no knowledge of hospital policies, despite mandatory training, and multiple online policy and procedures documents within the hospital. Only 15 participants demonstrated clear knowledge of the content of these policies, with 42 participants instead describing, in varying depth, how to access an interpreter, and ten having no knowledge at all. This did not differ across profession. Those who had recently used an interpreter were more like to know about procedures, but no more likely to know about the policy. A typical comment was "I'm sure there are policies and procedures on translator use to begin with uhm...but I haven't actually seen them myself or reviewed them".

\section{Discussion and conclusions}

\subsection{Discussion}

Our study examined factors influencing HPs' decision making about whether family members need an interpreter. We found the goals and beliefs HPs brought to the interactions with family members, as well as their assessment within the interaction of a family member's level of understanding, influenced their decision. Importantly, our study focussed on factors influencing whether they thought an interpreter was needed, not whether the family member received an interpreter. However, situational constraints, such as time, availability, and cost, 
that impact accessing an interpreter [18, 28], also influenced HPs' decision-making processes.

Underpinning HPs' decision making was their beliefs about the purpose of having an interpreter, which HPs stated was to enable them to communicate their serious or important information, so that family members understood. Their frame was a model of addressing the deficit of family members' understanding, rather than a frame of ensuring they dialogue with family members. Understanding was for most HPs a one-way process, where family members needed to understand HPs' messages, with few mentioning understanding what family members wanted to communicate to the health professional. Very few HPs reported an interpreter was needed to communicate regarding non-medical aspects of patient care or socioemotional needs, with both mostly not considered serious or important information. This did not differ by health profession. Thus, family-centred care was not a focus for HPs. This is consistent with Diamond et al [18] and also Jones et al [13], whose neonatal nurses described interactions with a culturally and linguistically diverse parent as focused on ensuring understanding, rather than a 2-way interaction, development of a shared identity, or a more interpersonal interaction. Moreover, while culturally competent care is a premise of family centred care [8], there was limited evidence in our study of HPs focusing on using interpreters to provide culturally competent care, with potential implications for parental well-being and patient care. Future research needs to consider both how LHLP parents perceive HPs focusing on the parent's understanding, the impact on parents, and the impact on quality of care. Finally, despite the focus on ensuring understanding, how much information needed to be understood differed across HPs. Future research should consider the implications for families where HPs focus on enough or complete understanding.

There was some evidence of racial or ethnic stereotypes influencing HPs' decision making. Although there is no research evidence to confirm their beliefs, some HPs expressed 
distrust of fathers from particular, mostly unnamed, racial or ethnic backgrounds. These stereotypes, consistent with community attitudes in Australia [42], were about fathers who were controlling of the mothers, and suggested a lack of trust in certain men as interpreters. Interestingly, a Dutch study found women trusted their children more than their husbands to interpret, suggesting HPs' perceptions may concur with some mothers' preferences, though more research is needed [43].

A further part of decision making was that within an interaction, HPs engaged in a process to assess the family member's understanding. Some HPs used a heuristic approach to their assessment, whereas others used more systematic processes. For those using a more heuristic approach, there was evidence of the representativeness heuristic, whereby family members with a particular physical appearance were assumed to be non-English-speaking. HPs also used fast and frugal evaluations, describing using a first impression of Englishspeaking ability, to make the decision. This is unsurprising given HPs are time poor and health systems have constrained budgets.

Our HPs described finding it hard to decide if an interpreter is needed. This may explain in part the variability between HPs in how they were making this assessment. Research on interpreter use has previously found HPs acknowledge under-utilizing interpreters, and instead are often "getting by" without an interpreter [18]. Our study demonstrates a number of HPs are also "getting by" in the process they use to assess whether an interpreter is needed, through the use of heuristics that met a given threshold for acceptability [35], and seeking enough rather than complete understanding.

Other HPs used more systematic processing, either alone or in combination with heuristics. These HPs demonstrated heightened awareness of the verbal and nonverbal cues of family members and used a range of techniques to test a family member's understanding. 
Future research should examine which techniques are most accurate at predicting whether a person needs an interpreter.

Surprisingly, there were few differences between health professions, as well as between monolingual and bilingual staff. There were suggestions interpreters are more for doctors than other HPs, and doctors were more often seen as both the person who could decide if an interpreter was needed and could decide as an individual rather than as a team. While not mentioned frequently these beliefs are contrary to hospital policies, although consistent with doctors having higher status and responsibility [44], or perhaps it is seen as part of their role. Some nurses perceiving themselves as having less responsibility for the decision may in part explain why nurses used heuristics more.

\section{Limitations}

Our study, while using the think aloud technique to mimic real-life decision making, is nonetheless still self-report. We have not examined what HPs do in practice, including whether those using a more heuristic vs systematic approach are more or less accurate in deciding when an interpreter is needed (acknowledging we know little about what is accuracy in interpreter utilisation decision-making). Future research could examine what happens when health professionals frame the decision as "when not to use an interpreter". In our study we also only examined how HPs decide if an interpreter is needed, not whether they use an interpreter. The decision to get an interpreter implicates other factors including their knowledge about how to get an interpreter. We did not gather specific details about their frequency and recency of interpreter use, to systematically examine their influence decision making. We also had a limited range of allied health professions and a small sample of doctors.

\subsection{Conclusion}


There is strong evidence use of interpreters improves the healthcare delivery for patients and families. However, our study demonstrates the decision about whether an interpreter is needed is one HPs find difficult, and there is no consistency in how they make this decision, with some using heuristics and others a more systematic approach. Moreover, HPs have beliefs about interpreter use (the focus on 1-way communication and HPs deciding when interpreter needed) that potentially limit the voice of family members, with implications for both family members and patients.

\subsection{Practice Implications}

HPs find the decision about whether an interpreter is needed difficult, and one for which they rarely received training or other assistance. There is also much variance in how they are making decisions, and what they consider to be 'enough' understanding. Thus, there is a need to provide skills training in working with LHLP families. As a part of this, it may be useful to develop a decision tool to assist hospital HPs in their decision making about whether an interpreter is needed, building on the tool developed for general practitioners in NZ [45]. At the same time, it is also important to ensure that HPs are aware of policies regarding interpreter use, including who is responsible for organising an interpreter, whether it should be a team decision, and for which health professionals and what information.

\section{CRediT roles}

Liz Jones: Conceptualization, Ethics application, Methodology , Formal analysis, Supervision, Writing- original draft

Nicola Sheeran: Conceptualization, Ethics application, Methodology , Data acquisition, Formal analysis, Writing- reviewing and editing

Rachyl Pines: Conceptualization, Ethics application, Methodology, Data acquisition, Formal analysis, Writing- reviewing and editing

Bradley Saunders: Conceptualization, Ethics application, Formal analysis, Writing- reviewing and editing 
Acknowledgements: We acknowledge the assistance of Dora Abbondanza in conducting interviews and undertaking transcription for this project, and Caelyn Jones for his role as research assistant.

I confirm all patient/personal identifiers have been removed or disguised so the person(s) described are not identifiable and cannot be identified through the details of the story.

\section{References}

1. Gooding JS, Cooper LG, Blaine AI, Franck LS, Howse JL, Berns SD. Family Support and FamilyCentered Care in the Neonatal Intensive Care Unit: Origins, Advances, Impact. Semin Perinatol. 2011;35(1):20-8.

2. Griffin T. Family-centered Care in the NICU. The Journal of Perinatal \& Neonatal Nursing. 2006;20(1):98-102.

3. Sheeran N, Jones L, Rowe J. The relationship between maternal age, communication and supportive relationships in the neonatal nursery for mothers of preterm infants. Journal of Neonatal Nursing. 2013;19(6):327-36.

4. Jones L, Taylor T, Watson B, Fenwick J, Dordic T. Negotiating care in the special care nursery: parents' and nurses' perceptions of nurse-parent communication. J Pediatr Nurs. 2015;30(6):e71e80.

5. Miles MS. Support for parents during a child's hospitalization: A nurse's guide to helping parents cope. Am J Nurs. 2006;103(2):62.

6. Cioffi J. Communicating with culturally and linguistically diverse patients in an acute care setting: nurses' experiences. Int J Nurs Stud. 2003;40:299-306.

7. Evans K. Nurses' perceptions of communicating with culturally and linguistically diverse parents in the neonatal nursery: A communication accommodation theory approach. Brisbane, Australia: Griffith University; 2015.

8. Hendson L, Reis, Misty D., \& Nicholas, David B. Health care providers' perspectives of providing culturally competent care in the NICU. Journal of Obstetric, Genecologic, \& Neonatal Nursing. 2015;44(1):17-27.

9. Australian Bureau of Statistics. Census Australia2011 [Available from: http://www.abs.gov.au/.

10. Paternotte E, Scheele F, Seeleman CM, Bank L, Scherpbier A, van Dulmen S. Intercultural doctor-patient communication in daily outpaitent care: relevant communication skills. Perspectives on Medical Education. 2016;5(5):8.

11. Cohen AL, Rivara F, Marcuse EK, McPhillips H, Davis R. Are language barriers associated with serious medical events in hospitalised pediatric patients? Pediatrics. 2005;116(3):575 - 9.

12. Hampers $L C, \&$ McNulty, Jennifer E. Professional interpreters and bilingual physicians in a pediatric emergency department. Arch Pediatr Adolesc Med. 2002;156:1108-13.

13. Jones L, Sheeran N, Lanyon H, Evans K, Dordic T. Nurses' perceptions of communicating with minority parents in a neonatal nursery: a communication accommodation theory aproach. Journal of Communication in Healthcare. 2018.

14. Jones L, Woodhouse D, Rowe J. Effective nurse parent communication: A study of parents' perceptions in the NICU environment. Patient Education and Counseling. 2007;69:206-12. 
15. Hyatt A, Lipson-Smith R, Schofield P, Gough K, Sze M, Aldride L. Communication challenges experienced by migrants with cancer: A comparision of migrant and English-speaking Australianborn cancer patients. Health Expect. 2017;20(5):10.

16. Department of Communities CSaDS. Queensland Language Services Policy. 2016.

17. Cioffi RNJ. Communicating with culturally and linguistically diverse patients in an acute care setting: nurses' experiences. Int J Nurs Stud. 2003;40(3):299-306.

18. Diamond L, Schenker, Yael., Curry, Leslie., Bradley, Elizabeth., \& Fernandez, Alicia. Getting by: underuse of interpreters by resident physicians. J Gen Intern Med. 2009;24(2):256-62.

19. Gerrish K. The nature and effect of communication difficulties arising from interactions between district nurses and Soth Asian patients and their carers. Journal of Advanced Nursing. 2001;33:11.

20. Gerrish K, Chau R, Sobowale A, Birks E. Bridging the language barrier: the use of interpreters in primary care nursing. Health and Social Care in the Community. 2004;12:7.

21. Karliner $L S$, Jacobs EA, Chen AH, Mutha S. Do professional interpreters improve clinical care for patients with limited English proficiency? A systematic review of the literature. Health Serv Res. 2007;42(2):727 - 54 .

22. Bischoff A, Denhaerynck K. What do language barriers cost? An exploratory study among asylum seekers in Switzerland. BMC Health Serv Res. 2010;10:248.

23. Jacobs EA, Sadowski, Larua S., \& Rathouz, Paul J. The impact of an enhanced interpreter service intervention on hospital costs and patient satisfaction. J Gen Intern Med. 2007;22(2):306-11.

24. Ngo-Metzger Q, Sorkin, Dara H., Phillips, Russell S., Greenfield, Sheldon., Massagli, Michael P., Clarridge, Brian., \& Kaplan, Sherrie H. Providing high-quality care for limited english proficient patients: The importance of language concordance and interpreter use. J Gen Intern Med. 2007;22:234-40.

25. Sterling LH, E Phung, H, Wong, K.Y. Client perceptions on the quality of the Queensland Health Interpreter Service. In: Health Q, editor. Brisbane Queensland: Queensland Government; 2012.

26. Ginde AA, Sullivan, Ashley F., Corel, Blanka., Caceres, Alfredo., \& Camargo Jr, Carlos A. Reevaluation of the effect of mandatory interpreter legislation on use of professional interpreters for ED patients with language barriers. Patient Education and Counseling. 2010;81:204-6. 27. Schenker Y, Perez-Stable, Eliseo J., Nickleach, Dana., \& Karliner, Leah S. Patterns of interpreter use for hospitalized patients with limited english proficiency. J Gen Intern Med. 2011;26(7):712-7.

28. Hsieh E. Not just "Getting by": Factors influencing providers' choice of interpreters. J Gen Intern Med. 2015;30(1):75-82.

29. Michalec B, Maiden, Kristin M., Ortiz, Jacqueline., Bell, Ann V., \& Ehrenthal, Deborah B. Providers' perceptions of medical interpreter services and limited english proficiency (LEP) patients: Understanding the "Bigger Picture". Journal of Applied Social Science. 2015;9(2):156-69.

30. Schenker Y, Pérez-Stable EJ, Nickleach D, Karliner LS. Patterns of Interpreter Use for Hospitalized Patients with Limited English Proficiency. J Gen Intern Med. 2011;26(7):712-7.

31. Lee LJ, Batal HA, Maselli JH, Kutner JS. Effect of Spanish interpretation method on patient satisfaction in an urban walk-in clinic. J Gen Intern Med. 2002;17(8):641 - 6.

32. Hilder J, Gray B, Dowell A, MacDonald L, Tester R, Stubbe M. 'It depends on the consultation': Revisiting use of family members as interpreters for general practice sonsultatonswhen and why? Aust J Public Health. 2017;23(3):7.

33. Gigerenzer G, Gaissmaier W. Heuristic Decision Making. Annual Review of Psychology. 2011;62(1):451-82.

34. Chaiken S. Heuristic versus systematic information processing and the use of source versus message cues in persuasion. J Pers Soc Psychol. 1980;39(5):752-66.

35. Gigerenzer G, \& Selten, R. Bounded rationality: The adaptive toolbox. MIT Press. 2002. 
36. Tversky A, Kahneman D. Judgement under uncertainty: Heuristics and biases. Science. 1974;185:8.

37. Steginga SK, \& Occhipinti, Stefano. The application of the Heuristic-Systematic processing model to treatment decision making about prostate cancer. Med Decis Making. 2004;24:573-83.

38. Statistics ABo. Migration Australia. Canberra, Australian Capital Territory2016.

39. Biggs SF, Rosman AJ, Sergenian GK. Methodological issues in judgment and decision-making research: Concurrent verbal protocol validity and simultaneous traces of process. Journal of Behavioral Decision Making. 1993;6(3):187-206.

40. Ericsson KA, Simon HA. How to Study Thinking in Everyday Life: Contrasting Think-Aloud Protocols With Descriptions and Explanations of Thinking. Mind, Culture, and Activity. 1998;5(3):17886.

41. Braun V, Clarke V. Using thematic analysis in psychology. Qualitative Research in Psychology. 2006;3(2):77-101.

42. Dandy J, Pe-Pua R. Attitudes to multiculturalism, immigration and cultural diversity: Comparison of dominant and non-dominant groups in three Australian states. International Journal of Intercultural Relations. 2010;34(1):13.

43. Zendedel R, Schouten BC, van Weert JC, van den Putte B. Informal interpreting in general practice: the migrant patient's voice. Ethn Health. 2018;23(2):16.

44. Watson B, Jones L, Hewett D. Accommodating health. In: Giles H, editor. Communication Accommodation Theory: Negotiating Personal Relationships and Social Identities Across Contexts. Cambridge: Cambridge University Press; 2016. p. 152-68.

45. Gray B, Hilder J, Stubbe M. How to use interpreters in general practice: the development of a New Zealand toolkit. J Prim Health Care. 2012;4(1):10.

$\underline{\text { Table 1. Participant demographic information }}$

Demographics

Age

$\underline{\text { Sex }}$

$\underline{\mathrm{M}}$

$\underline{\mathrm{F}}$

$\underline{\text { Profession }}$

Doctors

Nurses/midwives

Allied Health

$\underline{\text { Ward }}$

$\underline{\mathrm{NICU}}$

SCN

$\underline{\mathrm{PICU}}$

Pediatric Inpatient

$\underline{\text { Pediatric Outpatient }}$

Country of birth

$\underline{\text { Australia }}$

United Kingdom

South Africa

New Zealand, Malaysia, Philippines
Value

$\underline{\mathrm{M}=38.3(\mathrm{SD}=10.3)}$

$\underline{14.5 \%(\mathrm{n}=10)}$

$\underline{85.5 \%(\mathrm{n}=59)}$

$16 \%(\mathrm{n}=11)$

$\underline{74 \%(\mathrm{n}=51)}$

$10 \%(\mathrm{n}=7)$

$61 \%(\mathrm{n}=42)$

$7.2 \%(\mathrm{n}=5)$

$5.8 \%(\mathrm{n}=4)$

$2.9 \%$ each $(\mathrm{n}=2 \mathrm{each})$ 
United States, Singapore, Netherlands, India, Hong

Kong, Malta, Sri Lanka, Vietnam, Canada, South

Korea, Norway, Finland

$\underline{1.4 \% \text { each }(\mathrm{n}=1 \text { each })}$

Languages spoken

Monolingual

$\underline{73 \%(\mathrm{n}=50)}$

Bilingual or multilingual $\quad \underline{27 \%(\mathrm{n}=19)}$

$\underline{\text { Recent interpreter use }}$

$\underline{\text { Yes }}$

$\underline{46.4 \%(\mathrm{n}=32)}$

No

$53.6 \%(\mathrm{n}=37)$

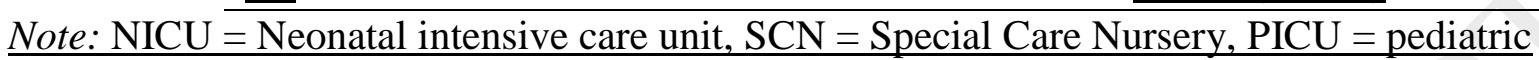
intensive care unit 
$\underline{\text { Table 2. Interview protocol }}$

1. What have you thought about when deciding whether or not a patient or patient's family member needs an interpreter/translator?

2. What do you think contributes to whether a person with LEP can understand what is said?

3. What do you think determines whether an interpreter is necessary?

4. What have you thought about when deciding who the most appropriate person to translate information is?

5. What contributes to whether a person is a suitable interpreter?

6. What do you think determines whether a person should not be used as an interpreter?

7. When you think of a person who needs an interpreter, what sort/kind/type of person or people do you think of?

8. What do you know about the pros and cons of using an interpreter?

9. Can you think of any issues in real life that could be obstacles and get in the way of accessing interpreters?

Specific probes:

10. Can you tell me your thoughts on using formal interpreters?

11. Can you tell me your thoughts on using a bilingual staff member as an interpreter?

12. Can you tell me your thoughts on using family members as interpreters?

13. Can you tell me your thoughts on using children as interpreters? 
Table 3. Themes and exemplar quotes.

\section{(i) Whose understanding?}

Parents understanding HP's message

HPs understanding parents'

message

Which parent/s

Which health professional

\section{(ii) Understanding what?}

Simple versus important or serious

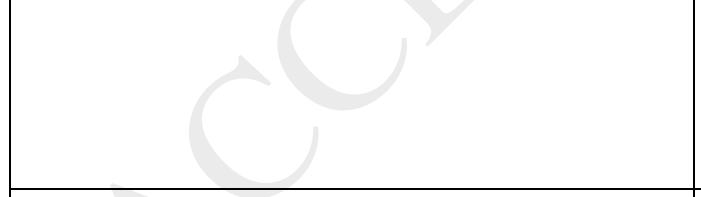

Medical care vs non-medical care

What is culturally appropriate care

(iii) How much understanding?

Complete vs enough "the parents, particularly the mum, should be able to understand what we're telling her" (110)

"their level of understanding uhmm if they're a language other than English" (109)

"I guess yeah how well I think they're understanding" (008)

"that the person feels maybe more welcomed and understood their voices may be heard" (010)

"pros are definitely increased understanding for both of us uhm because she can understand me and I can understand her and yeah much greater level of understanding" (008)

"we need to ensure that in the NICU environment for example both parents need to know what's going on" (108)

"in some ethnic origins you will get the partner he may be, the partner takes control and so he want's everything to go through him and you don't know what he is telling her, so that it is important that both the parents get the correct information, so that might sometimes determine why a husband can understand and speak English but his wife only partly understands we may need to get an interpreter involved." (108)

"In the past it has seemed that if they needed medical translating it would be done by, for, the medical team for their rounds but we haven't been having translators to assist the nurses communicate with the patients" (124)

"it's mainly those really...big decisions uhm kind of conversations like...you know obviously end of life stuff you would definitely have a translator involved in that kind of stuff or...but all the small decisions that you make bed bedside you probably couldn't continually call on a translator for those kind of judgements, work around it and use your hand and...other communication ways" (012)

"Get an informed consent which is very important in our work. Um to make things legal" (208)

"Depending on the type of situation and the acuity of the patient, so if the baby is pretty much healthy and just needs to learn to breastfeed or something like that, a lot of those things can be shown without words or with just a little bit of translation" $(002)$

"When the medical situation gets even more complicated and which also involve any cultural preferences and considerations, because sometimes we might not know or how to explain to them and let them understand, so maybe if they have the relevant language person that can speak to them, maybe they can make them understand better and we can get the idea better. That's what I think. Yeah cultural is quite different" (017)

"the family will...completely understand the situation" (002) 
1. Addressing socioemotional needs

"Anyone that can't speak English needs a translator for ANY condition. If a doctor's going to say, I'll take you temperature and they don't understand ...the thermometer shoving in their mouth" (212)

\begin{tabular}{|c|c|}
\hline & $\begin{array}{l}\text { "I think when we call in a translator it's often for the comfort for the family as well so someone whose going to be } \\
\text { able to, because these people they've got lots of emotions about what's going on and I'm sure it's a big relief when } \\
\text { they can speak to someone on the phone or in person in their own language so someone who is going to be } \\
\text { understanding and supportive of... who is able to validate the feelings of the clients of the family sorry or the } \\
\text { patient themselves" (126) }\end{array}$ \\
\hline \multicolumn{2}{|c|}{ 2. Who decides (whether an Interpreter is needed)? } \\
\hline Medical Professionals or Manager & $\begin{array}{l}\text { "Normally it's the medical team" (211) } \\
\text { "So if your boss thinks oh no that's fine you don't need to do that, then you don't." (208) }\end{array}$ \\
\hline Not the patient & $\begin{array}{l}\text { "maybe staff .. not believing that they need it when they ask for it...that's often a discussion that happens, 'they're } \\
\text { asking for a translator but they've understood me perfectly she could tell me where they lived or how they were } \\
\text { going today so I don't know why they're requesting that', I've heard that a few times" (119) }\end{array}$ \\
\hline \multicolumn{2}{|l|}{ 3. $\quad$ Assessing understanding } \\
\hline It is hard & $\begin{array}{l}\text { "sometimes when you've spent a little bit of time with someone and they ...lead you to believe a little bit that they do } \\
\text { understand, they'll keep up with you but you will realise that they don't actually know what you're... actually saying" } \\
(002)\end{array}$ \\
\hline Heuristics & $\begin{array}{l}\text { "I guess...the really obvious ... look at the demographic so sometimes African populations with very dark skin } \\
\text { you can kind of get a thing." (217) } \\
\text { "whether it is their second language" (003) }\end{array}$ \\
\hline Heuristic and systematic & $\begin{array}{l}\text { "usually when I've noticed a family from a different country, different ethnicity... I just say "oh are you from } \\
\text { Australia or recently from overseas. Sometimes when they speak to you, you'll notice they'll be missing words } \\
\text { when they speak English ...um well that's a good indicator they need a translator". (218) }\end{array}$ \\
\hline Systematic & $\begin{array}{l}\text { "I suppose whether or not they can hear what I'm saying and if my accent is an issue, the way that I'm explaining } \\
\text { things is an issue, and then if I give them a written form or a diagram, they can read that or if they can understand } \\
\text { what the diagram is, so ...I go mainly by their body language and by what their responses are. And if they're } \\
\text { showing no interest or no understanding and just look puzzled and then can't communicate much more than yes or } \\
\text { no then that sort of triggers me to um... ok...I don't think this person gets what I'm trying to say to them. So } \\
\text { whether or not they can take in what I'm saying, weigh things up and explain it back to me or explain their } \\
\text { decision back to me." (006) }\end{array}$ \\
\hline
\end{tabular}


4. Contextual factors

Time, Availability, Cost,

Knowledge of policies and

procedures
"Have we got time to wait" "The time it takes to organise an interpreter" (114)

"Difficult to organise" (220)

"They can be expensive to the organisation" (108) 